\title{
Development of radiofrequency ablation device for surgical hepatocellular carcinoma treatment in agreement with Brazilian standards
}

\author{
Gilvandson Costa Cavalcante ${ }^{1 *}$, Flávio Ferreira Lima ${ }^{2}$, Murilo Venturin ${ }^{3}$, Ricardo Pezzuol Jacobi ${ }^{1}$, \\ Suélia de Siqueira Rodrigues Fleury Rosa ${ }^{3}$ \\ ${ }^{1}$ Department of Mechanical Engineering, Technology College, University of Brasilia, Brasilia, DF, Brazil. \\ ${ }^{2}$ Department of Electrical Engineering, Technology College, University of Brasilia, Brasília, DF, Brazil. \\ ${ }^{3}$ Department of Electronic Engineering, Gama College, University of Brasilia, Brasília, DF, Brazil.
}

\begin{abstract}
Introduction: Many studies have been carried out on the main causes of hepatocellular carcinoma (HCC) to identify suitable diagnosis methods and treatments to combat the disease. However, high equipment costs and the lack of hepatic-ablation technologies manufactured in Brazil encourage the design and implementation of a solution based on normative principles. Methods: The analysis and description of equipment features were the first steps in defining the design requirements and constraints. Equipment functionalities were evaluated with three assays set by NBR-IEC 60601-2-2. Results: The assays of output control adjustment accuracy, output control adjustment monotonicity, and signal quality evaluation using the crest factor (CF) for the equipment showed thresholds superior to the aforementioned standard. Conclusion: State-of-the-art studies have contributed the equipment construction. The construction process was validated by assays to ensure the equipment functionality with raw materials at acceptable costs. The test results showed the superiority of the equipment in relation to the assays proposed by the standard NBR-IEC 60601-2-2. However, to convert the prototype into a commercial product, other analyses are necessary.
\end{abstract}

Keywords Accuracy, Monotonicity, Crest factor, Hepatocellular carcinoma.

\section{Introduction}

Hepatocellular carcinoma (HCC) is a type of liver cancer, and according to the World Health Organization (WHO) (Gomes et al., 2013), it is the second leading cause of death due to cancer, with approximately $70-85 \%$ deaths due to primary liver neoplasm (Gomes et al., 2013). The HCC is a malignant tumor that affects the main liver cells and occurs frequently worldwide, with more than one million cases per year (Audigier et al., 2015; Zheng and $\mathrm{Wu}, 2015)$. This cancer can develop owing to: excessive alcohol intake, infection with hepatitis B and/or $\mathrm{C}$, and induction of carcinogenic agents (Kikuchi, 2015).

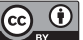

This is an Open Access article distributed under the terms of the Creative Commons Attribution License, which permits unrestricted use, distribution, and reproduction in any medium, provided the original work is properly cited.

How to cite this article: Cavalcante GC, Lima FF, Venturin M, Jacobi RP, Rosa SSRF. Development of radiofrequency ablation device for surgical hepatocellular carcinoma treatment in agreement with Brazilian standards. Res Biomed Eng. 2018; 34(2):115-126. DOI: $10.1590 / 2446-4740.07517$

*Corresponding author: Department of Mechanical Engineering, University of Brasília, Campus Darcy Ribeiro, Asa Norte, CEP 70910-900, Brasília, DF, Brazil. E-mail: gilvandson.costacavalcante@gmail.com Received: 27 October 2017 / Accepted: 15 April 2018
The anomaly diagnosis is performed with medical examinations using specialized equipment, such as ultrasound (special sound waves used in processes to examine organs inside the body), ultrasonography (the technique that produces images using sound waves to view structures inside the body), and computed tomography (Clavien et al., 2012; Cambridge..., 2018). However, when diagnostic failures occur due to hypervascularized or hypovascularized nodules, liver biopsy procedures are needed (Kikuchi, 2015).

Minimally invasive surgical treatments exist for this pathology, such as percutaneous ethanol injection (PEI), transarterial chemoembolization (TACE) and radiofrequency ablation (RFA) the last one being the most applied treatment worldwide (Haemmerich et al., 2001). There is evidence that RFA has advantages over PEI regarding HCC local control (Bouza et al., 2009).

Besides the aforementioned treatments, other techniques such as cryoablation (local tumor burning by freezing), high-intensity microwave ablation (similar to RFA; however, with frequencies of approximately $2450 \mathrm{MHz}$ ), and laser ablation (beams are confined through optical fiber) also exist. The treatment costs are illustrated in Table 1 (Pinheiro, 2016). 
Table 1. Internationally produced technologies used for liver cancer treatment (Pinheiro, 2016).

\begin{tabular}{ccc}
\hline Ablation Type & Equipment costs (USD) & Costs per section/electrode (USD) \\
\hline Cryoablation & $<190,000.00$ & $3,750.00$ (needle) \\
Laser & $30,000.00-75,000.00$ & $3,000.00$ (section) \\
Microwave & $65,000.00$ & - \\
Radiofrequency & $25,000.00$ & $800.00-1,200.00$ (electrode) \\
\hline
\end{tabular}

Table 2. Hepatic Ablation equipment manufactured internationally for cancer treatment.

\begin{tabular}{ccc}
\hline Model (Producer) & Production & Registry \\
\hline Barrx (COVIDIEN LLC) & USA & 10349000492 \\
CTRASES (COVIDIEN LLC) & USA & 10349000494 \\
RFG3 (COVIDIEN LLC) & USA & 10349000494 \\
ATAKR PLUS (MEDTRONIC) & USA & 10339190444 \\
GENIUS (MEDTRONIC) & USA & 10339190466 \\
UltraCinch LP (ST. J. MEDICAL) & USA & 10332340256 \\
TactiSys Quartz (ST. J. MEDICAL) & USA & 10332340382 \\
M-3004 (RF MEDICAL CO. Ltd.) & South Korea & 80410900047 \\
VULCAN EAS (SMITH \& NEPHEW) & USA & 80804050089 \\
Quantum 2 (ARTHROCARE CORPORATION) & USA & 80804050177 \\
\hline
\end{tabular}

According to data provided by the National Sanitary Surveillance Agency (Agência..., 2017), there exists no equipment manufactured in Brazil, as shown in Table 2.

The goal of this study is the development of an ablative high-frequency prototype $(400 \mathrm{kHz})$ with a maximum power of $50 \mathrm{~W}_{\mathrm{RMS}}$. The prototype construction was performed in agreement with the NBR-IEC 60601-2-2 (Associação..., 2013) standard. To supply the production methodologies of a radiofrequency $(\mathrm{RF})$ signal generator circuit and power control circuit, the according voltage, current, and temperature measurements, and conditioning circuits were described. Additionally, to evaluate the equipment construction process and measurement calibration, the tests were developed regarding the output control adjustment accuracy, output control monotonicity adjustment, and sinusoidal signal quality. Thereby, the operation requirements and product security could be tested in order to allow product industrialization and to obtain an ANVISA license (Agência..., 2017).

\section{Methods}

\section{Ablation equipment features}

Figure 1 illustrates the block diagram of the prototype. Two operation modes exist: manual and automatic. In automatic mode, the protocols are preset, and in manual mode, the physicians can freely choose the start/end power parameters, power steps, procedure finalization time, and the maximum temperature threshold for procedure interruption. Additionally, the prototype allows RS232 serial communication to send electric current, voltage, and temperature transmission parameters to other devices. These data facilitate the evaluation of electrical characteristics in the ablation region to verify cellular-necrosis occurrence for temperatures greater than $50{ }^{\circ} \mathrm{C}$ (Haemmerich et al., 2001) or to identify the occurrence of roll-off via an increasing impedance, described by the tissue charring point (Alba et al., 2011).

Block (1) represents a $400 \mathrm{~W}_{\mathrm{RMS}}$-switched-mode power supply with a $110 / 220 \mathrm{~V}_{\mathrm{CA}}$-supply and a continuous $24 \mathrm{~V}_{\mathrm{DC}}$-output voltage with efficiency greater than $80 \%$. In addition, it has protection against opposite surge currents, short circuits, overload, and overheating. The output of the power supply is connected to two voltage regulators (LM7805 and LM7812, Fairchild, California, USA) (Fairchild..., 2008) with outputs of 5 and $12 \mathrm{~V}_{\mathrm{DC}}$. The output is fully shielded to avoid interference.

Block (2) is responsible for $400 \mathrm{kHz}$-sine wave signal generation and prepared to receive a power adjustment signal from the control board of 5-50 $\mathrm{W}_{\mathrm{RMS}}$. In addition, this board contains circuits to measure the voltage and current applied to the liver during the procedure. These measurements are used to calculate power and impedance according to the ablation evolution.

Block (3) is the power control board that adjusts the voltage level provided by the power supply to the $\mathrm{RF}$ generator board to define the power applied to the liver. The adjustable output levels of the control board measure 1.2-24 $\mathrm{V}_{\mathrm{DC}}$, with sufficient electric current to ensure $50 \mathrm{~W}_{\mathrm{RMS}}$. The voltage variations are controlled by the Raspberry PI III using ADC/DAC converters and proportional-integral-derivative (PID) controllers through the software implemented on it.

Block (4) performs the digital equipment management conducting voltage, current, and temperature measurements. 


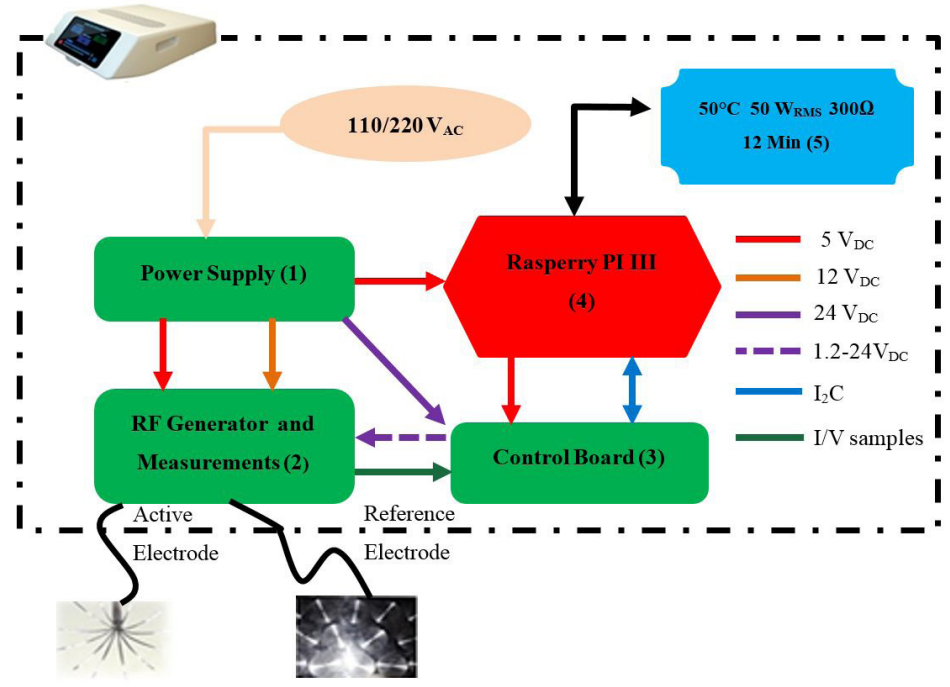

Figure 1. Block diagram of Measurement setup. Block (1) represents the power supply with output voltages of 5, 12, and $24 \mathrm{~V}_{\mathrm{DC}}$. Block (2) is the radiofrequency (RF) generator board and voltage/current measurement circuit. Block (3) refers to the power control board applied to the liver in combination with measuring circuits. Block (4) comprises the digital control equipment that sends and receives touch screen information, represented by block (5).

The Raspberry PI adjusts the power applied to the liver and sends the measured information to the touch screen, block (5).

Block (5) represents the user interface block. Power, impedance, temperature values, and procedure time are displayed on the screen. In addition, it provides the input values to configure the prototype operation.

\section{RF generation board and data acquisition}

Figure 2 shows a block diagram of the RF generator board with the corresponding voltage and current measurement stages. The circuit is composed of: 1 ) astable oscillator adjusted to a $400 \mathrm{kHz}$-frequency; 2) non-overlapping clock (NOC); 3) voltage driver; 4) frequency inverter circuit; 5) protection filter against voltage return from RF generator to control board; 6) a filter to convert the square wave into a sinusoidal wave; 7) peak detector circuit for voltage measurements; 8) operational amplifier to maintain $5 \mathrm{~V}_{\mathrm{DC}}$-maximum excursion; 9) filter to avoid interference in the voltage measurement circuit; 10) peak detector circuit for current measurement; 11) operational amplifier to maintain $5 \mathrm{~V}_{\mathrm{DC}}$-maximum excursion; 12) filter to avoid interference in the current measurement circuit.

\section{NOC}

The output signal of the astable oscillator is delivered to the NOC circuit, which divides the signal into two parts with phases shifted by $180^{\circ}$. In addition, the NOC circuit negates the possibility that the square wave might appear as a trapezoidal wave. This procedure is necessary to avoid simultaneous switching of power transistors, which are trigged by the frequency inverter input. In this setup, two combinational circuits built with NAND ports (74HC00-Philips, Amesterdã, Netherlands) (Philips..., 1997) and nine Schmitt-Trigger logic inverters (74HC14-Nexperia, Nijmegen, Netherlands) (Nexperia..., 2017) were applied. The construction topology of this circuit is described in (Kavak, 2004).

\section{Voltage drive}

The voltage driver (IR2110-International Rectifier, California, USA) (International..., 2005), Figure 2, block 3 , acts as a signal amplifier for signals originating from the NOC circuit (5-12 $\left.\mathrm{V}_{\mathrm{DC}}\right)$. After amplification, the signals are used to switch the MOSFET power transistors (IRF540-Vishay Siliconix, California, USA) (Vishay..., 2013a), which are connected to the input of the frequency inverter circuit. Thus, it is possible to ensure that the electric current coming from the control circuit is sufficient to provide the power required for ablation.

\section{Frequency inverter}

The signal from the voltage driver (IR2110), Figure 2, block 3 , is sent to the frequency inverter circuit, which amplifies it. The frequency inverter is composed of two MOSFETs (IRF540) with drains connected to each terminal of the power transformer and the source connected to ground. The transformer has a center tap connected to a second-order Butterworth filter, and to 


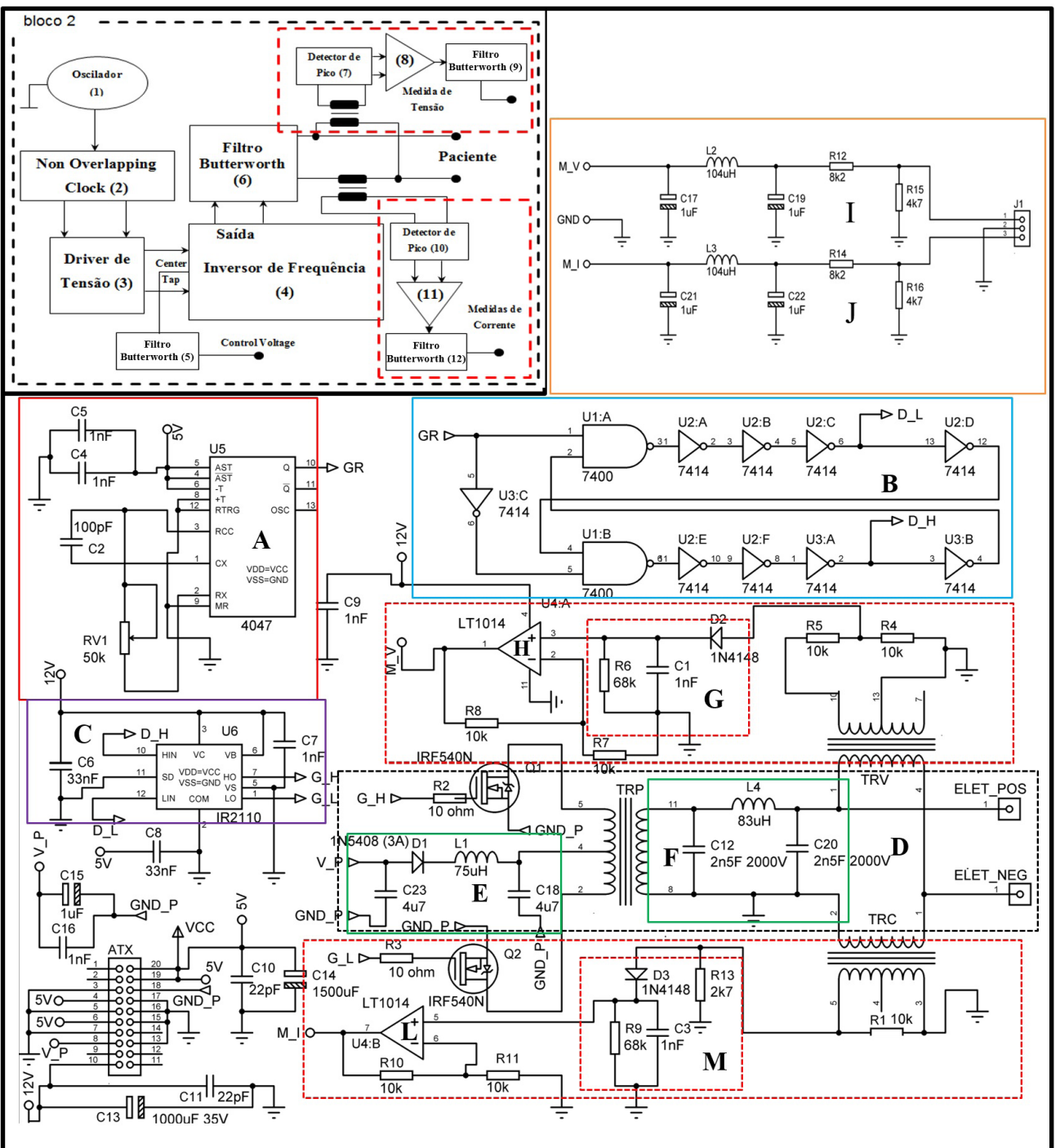

Figure 2. In this figure, the blocks $(1=\mathrm{A}),(2=\mathrm{B}),(3=\mathrm{C}),(4=\mathrm{D}),(5=\mathrm{E}),(6=\mathrm{F}),(7=\mathrm{G}),(8=\mathrm{H}),(9=\mathrm{I}),(10=\mathrm{M}),(11=\mathrm{L})$ and $(12=\mathrm{J})$ represent respectively, the oscillator, the Non-Overlapping Clock (NOC), voltage driver, frequency inverter, filter Butterworth, filter Butterworth, peak detector, operational amplifier, filter Butterworth, peak detector, operational amplifier and filter Butterworth.

a diode (1N5408-Vishay Siliconix, California, USA) (Vishay..., 2013b) to avoid voltage return caused by a discharge of the electromagnetic field stored in the transformer. It is observed that when the voltage applied to the central tap increases, the amplitude of the square signal and consequently the power increase. This circuit has a theoretical efficiency of $75 \%$ (Frenzel, 2015).

\section{Butterworth filter design}

The PI topology of the filters presented in Figure 2 has distinct functions in the RF generator circuit; all filters are second-order and low-pass band. In Figure 2, the filter (block 5) prevents a voltage return from the power transformer to the control circuit. This filter contains a
$73 \mu \mathrm{H}$-inductor and two capacitors of $4.7 \mu \mathrm{F}$, and has a cutting frequency of $8.597 \mathrm{kHz}$. In block (6), in addition to converting the square wave into a sinusoidal wave, the circuit further matches the impedance of the frequency inverter to that of the liver. It has an $83 \mu \mathrm{H}$-inductor and two capacitors of $2.5 \mathrm{nF}$, and has a cutting frequency of $349.39 \mathrm{kHz}$. Moreover, in blocks (9) and (12), the filter removes high frequency components from the output signals of the voltage and current circuits of the liver. It possesses a $104 \mu \mathrm{H}$-inductor and two capacitors of $10 \mu \mathrm{F}$, and has a cutting frequency of $4.94 \mathrm{kHz}$.

For the construction of a Butterworth filter with PI topology, the circuit transfer function was extracted. The Laplace transform and the Kirchhoff current laws 
were applied to obtain Equation 1 (Alexander and Sadiku, 2008). According to the modeling, capacitance $C_{12}$ does not contribute to the system behavior when the input signal is applied to this capacitor, Figure 2, block F. After completion of the modeling process, Equation 1 must obey the parameterization rules of Equation 2, which represents the standard function of a second-order low-pass filter (Vernetti, 2003).

$$
\begin{aligned}
& H(s)=\frac{1}{L_{4} C_{20} S^{2}+\frac{L_{4}}{R} S+1}=\frac{\left(1 / L_{4} C_{20}\right)}{S^{2}+\frac{1}{R C_{20}} S+\frac{1}{L_{4} C_{20}}} \\
& H(s)=K \frac{b_{i}}{S^{2}+a_{i} S+b_{i}}
\end{aligned}
$$

Owing to the use of low-pass filters, the quality factor $\left(Q_{\mathrm{p}}\right)$ was determined with Equation 3. The higher the quality factor, the closer are the poles to the imaginary axis in the complex plane, thereby increasing the selectivity of the filter (Vernetti, 2003).

$$
Q_{p}=\frac{\sqrt{b_{i}}}{a_{i}}=\frac{R C_{20}}{\sqrt{L_{4} C_{20}}}=R \sqrt{\frac{C_{20}}{L_{4}}}
$$

Considering the characteristic behavior of a liver ablation region, which varies its impedance as a function of rising temperature, $Q_{\mathrm{p}}$, specified in Figure 2, block (6), was evaluated. This way, a resistive load of $50 \Omega$ was established as a reference for the initial calculation of $Q_{\mathrm{p}}$ with Equation 3, because the value of $Q_{\mathrm{p}}$ for this load is $\leq 0.707$ (Vernetti, 2003). Using the resistive, capacitive, and inductive values presented for the filter in Figure 2, block (6), the estimated value was 0.27. This value means that the highest output voltage $\mathrm{V}_{\text {out }}$ occurs when the angular frequency is $\omega=0$ for a DC voltage (see Equation 5 (Vernetti, 2003)). However, this value undergoes changes during ablation and, therefore, the analysis of this variation was considered in the tests. Regarding the other filters, the impedances at the outputs are fixed and larger than $50 \Omega$. This results in higher quality factors than that calculated for the filter of Figure 2, block (6).

The calculation of the maximum power necessary for ablation depends on the maximum amplitude of $V_{\text {out }}$ of the filter in Figure 2, block (6), applied at the location of liver ablation. To perform the described analysis, it was considered that $H(\mathrm{~s})=V_{\text {out }} / V_{\text {in }}$ (Equation 1) and the derivative of $V_{\text {out }}$, as a function of $\omega$, was equated to zero for $s=j \omega$. The result obtained from this mathematical manipulation together with Equation 3 corresponds to Equation 5 (Alexander and Sadiku, 2008).

$$
\omega_{p}=\sqrt{b_{i}}=\frac{1}{\sqrt{L_{4} C_{20}}}
$$

$\frac{d}{d \omega}\left|\frac{1}{-\omega^{2} L_{4} C_{20}+\frac{J \omega L_{4}}{R}+1}\right|=0, \omega=\omega_{p} \sqrt{1-\frac{1}{2 Q_{p}^{2}}}, Q_{p} \geq 0.707$

With Equation 5, it was possible to evaluate the two situations. Firstly, when $Q_{\mathrm{p}} \leq 0.707$, the maximum $\mathrm{V}_{\text {out }}$ will occur for $\omega=0$ (case cited above). Secondly, when $Q_{\mathrm{p}}>>1$, a voltage peak occurs at $\omega$, Equation 5 (Vernetti, 2003).

Further, $Q_{\mathrm{p}}$ indicates that the filter is more selective and must be considered in the monotonicity adjustment because the power peaks rise according to the quality factor function.

The inductor filters in Figure 2, blocks (5) and (6), were constructed with an air core, and the circuit capacitors of block (5) had a threshold voltage of $240 \mathrm{~V}$ (commercial value) due to the applied maximum voltage of $24 \mathrm{~V}_{\mathrm{DC}}$. In block (6), the filter capacitors had a threshold of $2000 \mathrm{~V}$ to avoid device heating. The other filters use ferrite core inductors and electrolytic capacitors with thresholds of $25 \mathrm{~V}$.

\section{Power transformer design}

The power transformer belongs to the frequency inverter circuit in Figure 2, block (4). During its development, precautions were taken to avoid Joule heating that would result in power losses. These losses are only perceptible when the temperature is higher than $80^{\circ} \mathrm{C}$. In addition, laminar transformers were avoided due to high-power losses by Foucault currents when the power transformer is operating at high frequencies (Xiaojing et al., 2016).

Therefore, during the transformer development, the turns ratio between primary and secondary coils was $1: 10$. This ratio was designed to achieve the $50 \mathrm{~W}_{\text {RMS }}$. The power transformer was installed in an in vitro assay with swine liver. The turns ratio was chosen according to the acquisition facility on the national market of switched-mode power supplies. In this study, the applied ferrite core specified the magnetic flux density according to the fabrication guidelines (Thornton Eletrônica Ltda) (Thornton..., 2018) and in agreement with Equations 6 and 7.

$$
V_{R M S}=\sqrt{P Z}
$$

$$
B=\frac{V_{R M S}}{4 f N A_{e}}
$$

Equation 6 is used to calculate the RMS voltage $\left(\mathrm{V}_{\mathrm{RMS}}\right)$ applied to the liver, depending on the maximum power $P$, with additional $50 \%$, and on the estimate of 
the impedance maximum $Z$ when roll-off occurs during ablation. The obtained value is used in Equation 7 to determine the magnetic flux density (B) considering a frequency (f) of $400 \mathrm{kHz}$, a turn number of 3 in the primary coil $(N)$ for a center-tap connected coil, and the ferrite core's effective area $\left(A_{\mathrm{e}}\right)$.

The inductance factor $\left(A_{\mathrm{L}}\right)$ is defined in Equation 8: $N$ represents the turn number of 3 for a center tap-connected coil and $L$ the transformer's primary coil inductance. These two parameters are used for impedance matching realized by the PI circuit construction, Figure 3, using electronic components of the national market.

$$
A_{L}=\frac{L}{N^{2}}
$$

The employed transformer core was a ferrite model, NEER-28/17/12-2200-IP12E (Thornton Eletrônica Ltda) (Thornton..., 2018). Regarding an operation at high frequencies with acceptable losses, the ferrite core should not possess air gaps nor $90^{\circ}$-corners.

The inductance factor $\left(A_{\mathrm{L}}\right)$ of the ferrite core determines $L$ of the transformer's primary and secondary coil. However, the coil inductance is negligible when the coil does not comprise this material. Here, the inductance of the transformer's primary coil was measured with ferrite, using an ICEL RLC bridge, L301, with $17 \mu \mathrm{H}$. However, as the ferrite possesses $A_{\mathrm{L}}=2.2 \mu \mathrm{H}$, according to the data sheet, the inductance value is $19.8 \mu \mathrm{H}$. Despite the difference between the value specified by the manufacturer and the measured one, there was no impact on the equipment development.

\section{Current, voltage and temperature measurement circuits}

These circuits were used to evaluate the biophysical parameters during ablation. Power and impedance are the main liver incident power control parameters.

The transformers used to measure current and voltage were developed according to the power transformer construction methodology. However, the current measurement transformer has two turns with a $2.5 \mathrm{~mm}$-wire gauge to ensure smaller efficiency losses under heating during measurement.

To measure the current, the transformer is connected in series through an electrode inserted into the patient ablation area, and parallel for a voltage measurement device. The samples obtained from the current transformer flow through a resistor, which facilitates the current to voltage conversion. The signal amplitudes are conditioned by peak detectors and standardized through amplification and filtration. The signals are further sent to an ADC (ADS1115 -Texas Instruments, Dallas, EUA) (Texas..., 2018) converter input that is part of the control circuit.

The temperature measurement is performed by a $100 \mathrm{k} \Omega$-thermistor (PTC). The measurement circuit was built connecting a $4 \mathrm{k} \Omega$-resistor in series to the thermistor. The voltage values provided by the divisor are collected by the ADC (ADS1115).

The discrete values are sent from the ADC to the Raspberry PI through a serial communication bus using the $\mathrm{I}_{2} \mathrm{C}$ protocol. The ADC and DAC (PCF8591-Nexperia, Nimega, Netherlands) (Nexperia..., 2013) converters share the same bus. The manufacturer recommends the insertion of an electrolytic $10 \mu \mathrm{F}$-capacitor into the bus to avoid interference and spurious noise caused by high

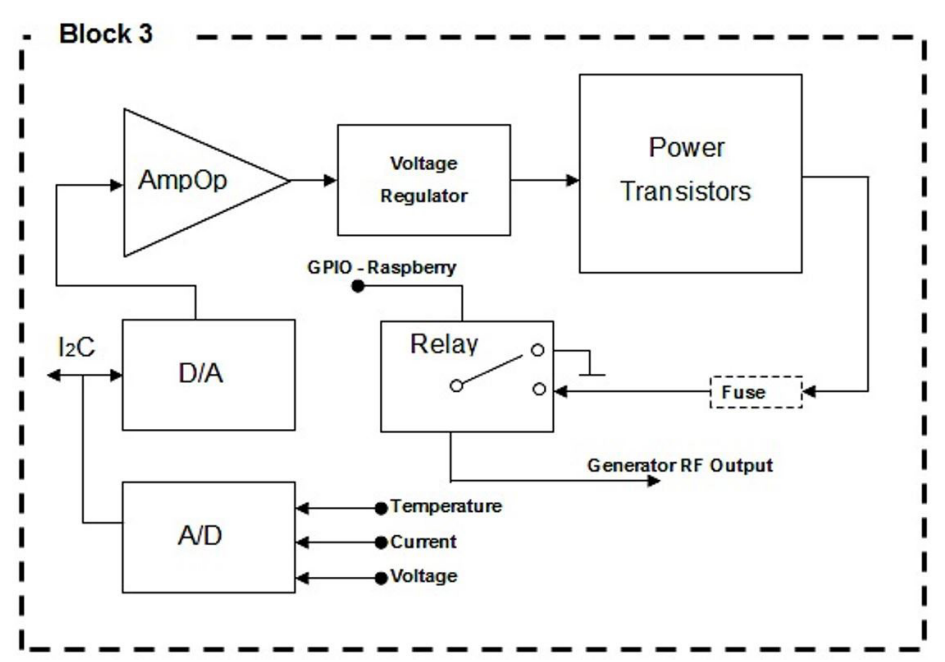

Figure 3. This diagram represents the circuit responsible for the power control. The DAC converter's output voltage is amplified and transmitted to the RF generator and measurement of block (2), Figure 1. 
frequencies. The cables through which these protocols are transmitted were shielded to increase their immunity against such interference.

\section{Power control board}

In the liver tumor ablation process, the RF generator board is controlled by voltage variations in the transformer's center tap provided by the control circuit shown in Figure 3.

The power control board is the interface between Raspberry PI III and RF generator block. The following components were used for the board design: DAC (PCF 8591); ADC; operation amplifier (Op-amp, LT1014-Linear, California, USA) (Linear..., 1990); adjustable voltage regulator (LM317-Texas Instruments, Dallas, USA) (Texas..., 2017a); bipolar power transistors; bipolar transistor (BC556-ON Semiconductor, Colorado, USA) (On Semiconductor, 2007); fuses and relay.

The DAC converter receives data from the Raspberry $\mathrm{PI}$ via the $\mathrm{I}_{2} \mathrm{C}$ communication protocol. This data represents a discrete analog control signal with 8-bit-resolution. The analog output of the device is amplified by an AmpOp that has its output connected to the transistor base (BC556) to adjust the voltage for the voltage regulator (LM324-Texas Instruments, Dallas, USA) (Texas..., 2017b) in order to obtain a voltage range between 1.2-24 $\mathrm{V}_{\mathrm{DC}}$.

The voltage regulator output excites the base of three power transistors through $150 \Omega$-resistors with the goal to control the current that flows through these transistors when the drain is connected to the RF generator board. Without the resistors, the current would not circulate evenly, thereby inducing heat in each transistor.

The transistors provide enough current to supply the necessary power to the ablation process through the RF generator board. This control board, shown in Figure 3, has relay and a fuse protection in the output signal. The relay is triggered by GPIO (input/output port of Raspberry PI). The output voltage in this circuit is only released by a digital management command in the relay.

The ADC (ADS1115) converter has 4 analog input channels. Hence, three of them are used for reading current, voltage, and temperature. The last input channel is not employed. All channels have a maximum excursion of an input voltage of $0-5 \mathrm{~V}$.

\section{Digital management}

Digital management is conducted by the Raspberry PI III, which uses an ARMv8 quad-core BCM2837 processor with a clock frequency of $1.2 \mathrm{GHz}$ and Wi-Fi connection. The embedded system that performs ablation functions was developed in Python. Using this programming language, it was possible to develop the graphic interface, serial communication using the transmission protocols $\mathrm{RS} 232$ and $\mathrm{I}_{2} \mathrm{C}$, temperature stop or timeout, and power control through the proportional-integral-derivative (PID).

\section{Voltage, current and temperature measurement calibration}

In this process, for the calibration of voltage and current, a digital oscilloscope (Tektronix, TDS1001B), with a $40 \mathrm{MHz}$-bandwidth and a sampling rate of $500 \mathrm{MS} / \mathrm{s}$ was applied. The calibration curves for these measurements were obtained using purely resistive loads that could support until $100 \mathrm{~W}_{\mathrm{rms}}$, although only $50 \mathrm{~W}_{\mathrm{rms}}$ was supplied by the equipment, in accordance with NBR-IEC 60601-2-2 (Associação..., 2013). In the calibration process, the electrode impedance was also considered. At the same time, the data derived from the ADC converter was stored in the Raspberry. Then, using MATLAB software, it was possible to obtain the calibration functions concerning voltage and current using linear regression.

The calibration of the temperature measurement, using PTC, in the ablation region was performed using the Raspberry PI. For this purpose, a Python program was developed to capture the analog information of the temperature sensor and to store them with a resolution of one degree in an Excel spreadsheet. A water container was used and then the temperature was changed. In parallel a DT858L multimeter that has a type $\mathrm{k}$ thermocouple with an error of $0.8^{\circ} \mathrm{C}$ was used, collecting the same information. With both data and using the same process applied at voltage and current calibration, the calibration function about temperature was obtained.

\section{Bench tests}

During the ablation equipment development phase, the equipment was calibrated using the RMS values for voltage and current. However, to obtain the declared power, in compliance with the standard NBR-IEC 60601-2-2 (Associação..., 2013), the RMS power was applied in conjunction with the CF obtained at each point of the curve. These adjustments consider small distortions of the equipment's output signal to avoid performing a rough approximation by multiplying the value by $\sqrt{2}$. To guarantee test robustness, the temperature of the power transformer was monitored using a GM320 digital pyrometer and maintained at approximately $50{ }^{\circ} \mathrm{C}$. Thus, it was possible to verify that the power transformer operated within the technical characteristics defined during development. The temperature was required to not exceed $80^{\circ} \mathrm{C}$ in order to avoid power loss due to excess temperature.

The test bench was prepared in agreement with NBR-IEC 60601-2-2 (Associação..., 2013). The table was $1 \mathrm{~m}$ in height and is made from wood, put on top of an aluminum board, and connected to a neutral outlet. The load used in this experiment has inductive components. However, the impedance phase does not 
exceed $1^{\circ}$, thereby being much lower than the maximum value of $8.5^{\circ}$ recommended by the IEC 60601-2-2 (Associação..., 2013). The values were evaluated with Equation 9 such that $X_{\mathrm{L}}$ is equivalent to the inductive reactance. The parameter $F$ is the standard frequency of $1 \mathrm{kHz}$ and $L$ the resistor's intrinsic inductance. The impedance phase $\theta$ was calculated according to Equation 10.

$$
X_{L}=j 2 \pi F L
$$

$$
\theta=\arctan \left(\frac{\left|X_{L}\right|}{|R|}\right)
$$

In addition, the following ambient conditions were used to employ the proposed test benches: a temperature of $22.3{ }^{\circ} \mathrm{C}$, a relative humidity of $52 \%$, and an atmospheric pressure of $1021.6 \mathrm{hPa}$.

A stainless-steel plate measuring $16.0 \times 11.9 \mathrm{~cm}$ was used as neutral electrode connected to the equipment's ground, according to the NBR-IEC 60601-2-2 (Associação..., 2013). For the RF output, an active umbrella-type nitinol electrode was developed and validated (Pinheiro, 2016). The loads were integrated between the active and neutral electrodes for the assays.

\section{Output control adjustment accuracy}

To validate the measurements performed in the assays, the values obtained by the ablation equipment were compared to those measured with the oscilloscope. In the evaluation of the measurement accuracy of the ablation equipment, a load of $100 \Omega$ was adopted, according to the NBR-IEC 60601-2-2 (Associação..., 2013). The ablation equipment was adjusted manually with an initial power of 5 and a final power of $50 \mathrm{~W}_{\mathrm{RMS}}$. The power increase was programmed in ten steps of $5 \mathrm{~W}_{\mathrm{RMS}}$ for ten minutes. Each second, the power control dataset was automatically stored in the spreadsheet. The output power dataset obtained by the oscilloscope was stored similarly. Through these datasets, it was possible to verify the error of the output control adjustment accuracy.

\section{Output control monotonicity adjustment}

For conformity, the mean and maximum thresholds of the - in the liver ablation region applied - power were evaluated. With the tests, it was possible to evaluate the existence of power peaks and to verify whether the applied power remains constant within the error range defined by NBR-IEC 60601-2-2 (Associação..., 2013), regarding the declared loads defined for the equipment.

The active RF electrode cable is $0.5 \mathrm{~m}$ away from the reference electrode cable. To comply with NBR-IEC 60601-2-2 (Associação..., 2013), the resistors used in the assays need to have a threshold larger than $50 \%$ of the maximum power provided by equipment with a load of $50 \mathrm{~W}_{\mathrm{RMS}}$.

\section{Signal analysis using Crest Factor (CF).}

The CF indicates the wave distortion degree in relation to the sine wave in the time domain (Richman, 2002). Regarding the identification of a pure sine wave, i.e., no distortion, the CF is $\sqrt{2}$ (Richman, 2002). Thus, the evaluation of this parameter in the ablation prototype is important to verify whether the ablation equipment's output signal is close to the pure sine wave's signal. The closer the obtained value is to 1.414 , the greater is the burn efficiency (Schneider-Junior and Abatti, 2005).

$C F=\frac{V_{P}}{V_{R M S}}=\frac{R I_{P}}{R I_{R M S}}=\frac{I_{P}}{I_{R M S}}$

In order to evaluate the $\mathrm{CF}$, the same methodology was applied for the load association in the monotonicity test. The peak voltage $\left(V_{\mathrm{p}}\right)$ and $V_{\mathrm{RMS}}$ measurements were performed with a digital oscilloscope, considering a maximum power of $50 \mathrm{~W}_{\mathrm{RMS}}$ or approximately $100 \mathrm{~W}$ of peak power.

\section{Results}

All the performed tests had an equipment output signal with a frequency of $406.05 \mathrm{kHz}$ and a standard deviation of $1.25 \mathrm{kHz}$. Because the preset frequency was $400 \mathrm{kHz}$, this resulted in a variation of $\pm 1.51 \%$.

\section{Control adjustment accuracy test}

The results of the control adjustment accuracy tests are shown in Figure 4, according to NBR-IEC 60601-2-2 (Associação..., 2013). The curve shows the peak power obtained in the load through measurements using a digital oscilloscope and the power measured in the equipment proposal that was displayed on the touch screen. The relation between the values obtained using the oscilloscope and equipment (in percentage points) is shown in Figure 4.

\section{Monotonicity assay}

In this assay, the mean and maximum power thresholds applied in the loads defined due to estimated variation of the liver impedance in the ablation process were evaluated (see points shown in Figure 5).

In this evaluation, it was verified that the percentages of output power errors measured by the oscilloscope in relation to those obtained by the ablation equipment at the critical points highlighted in the curve (Figure 5) are well below that specified by NBR-IEC 60601-2-2 (Associação..., 2013), that is $20 \%$. The highest error found was $7.97 \%$.

\section{CF measurement}

Figure 6 shows the results obtained from the ablation signal. This curve was evaluated, with the CF of 1.414 of a pure sine wave as reference. Thus, the error percentage represented by the green curve shows how closely the measured signal matches the pure sine wave signal. 


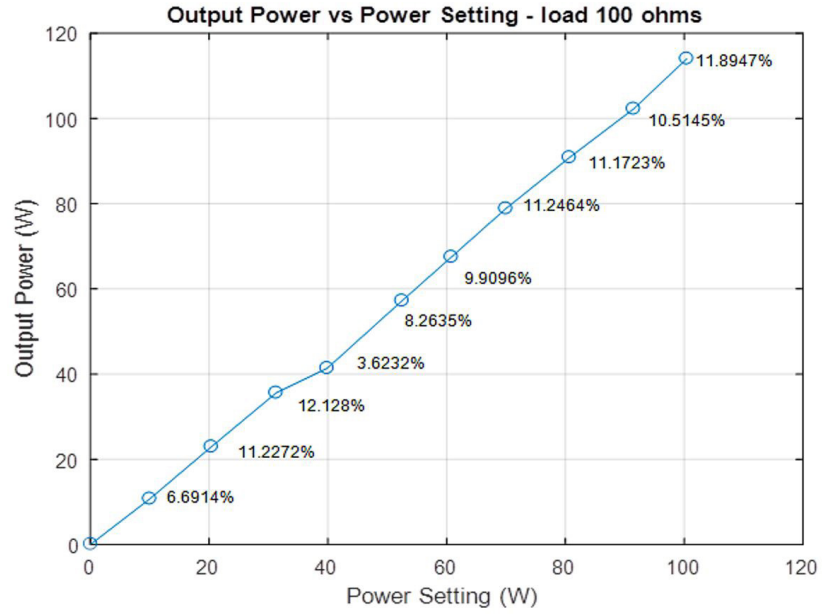

Figure 4. In this graph, it is possible to evaluate the measurements using a digital oscilloscope and the equipment proposal. The errors range from $3.623-12.128 \%$. The declared output power values of the equipment are on the Y-axis and the power values established by the equipment to be supplied at its output are on the $\mathrm{X}$-axis.

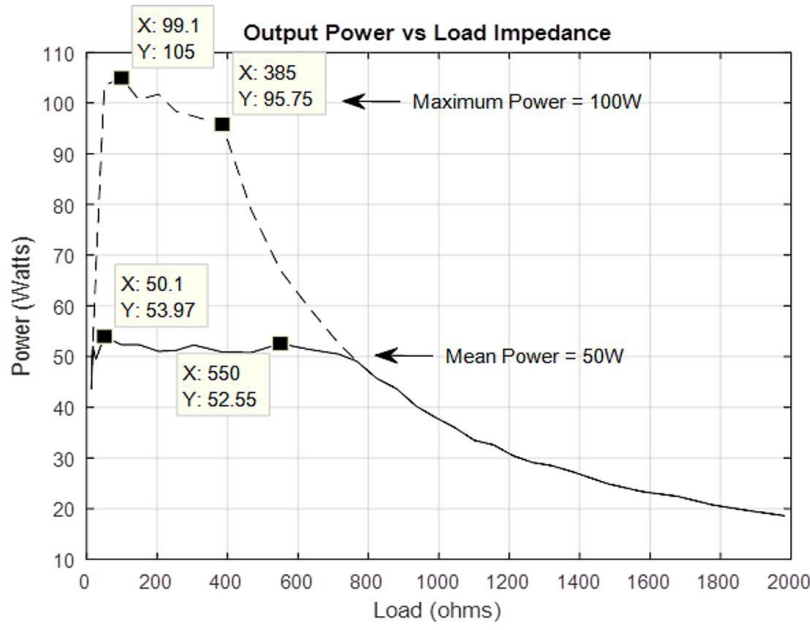

Figure 5. Monotonicity assay. In this figure, the values of the critical points are shown. The X-value refers to the non-inductive resistive load at the instant of power application and the Y-value to the mean and maximum declared power of the equipment.

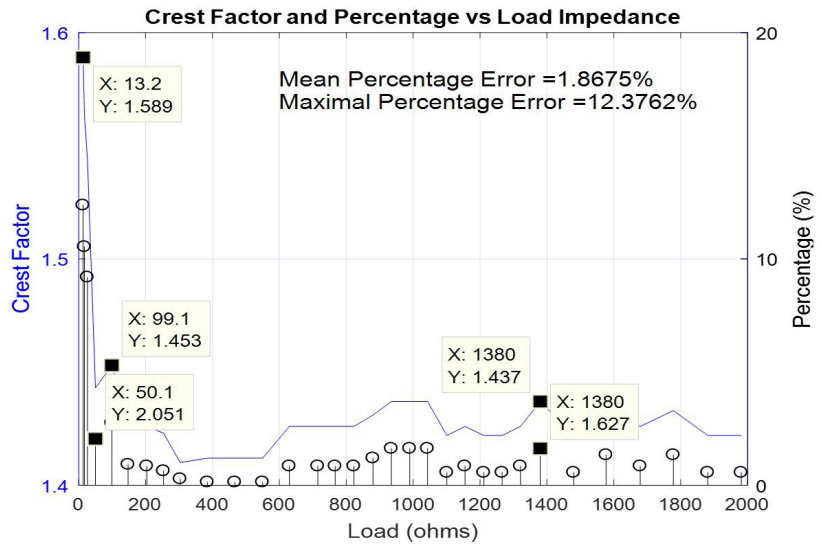

Figure 6. $\mathrm{CF}$ evaluation for the declared loads. The $\mathrm{X}$-values represent the load values for both curves, and the Y-values in the blue curve represent the $\mathrm{CF}$. The green axis illustrates the $\mathrm{CF}$ percentage. 


\section{Discussion}

State-of-the-art surveys motivated the elaboration of this prototype for hepatic ablation. The development stages and details on the RF generation board, power control, and measurement circuits with their respective calibrations were described. The successful operation of each proposed circuit was validated with assays.

In the literature, there are no details of other equipment available for comparative analysis. Because of this, standard NBR-IEC 60601-2-2 (Associação..., 2013) was used for conformity assessment of the equipment. The innovation is defined by the fact that there is no product available in Brazil certified by ANVISA (Agência..., 2017).

Regarding the output control adjustment accuracy tests, it can be seen that the maximum control power error in relation to the declared output power measured $12.128 \%$. The value is below the defined error of 20\% NBR-IEC 60601-2-2 (Associação..., 2013). Thus, the result shows that the equipment proposal works in accordance with NBR-IEC 60601-2-2 (Associação..., 2013) in relation to this type of test. This power control is essential to prevent hurting patients and to reduce the carbonization rate, ensuring a larger firing diameter and maximum efficiency in fighting the disease. Besides, the current and voltage measurements conducted by the equipment in the liver revealed to be reliable. These parameters give information on liver power and impedance values. The precision of these measurements, besides ensuring efficient control of the power applied to the liver, facilitates procedure interruption via impedance measurements in a coherent way, thereby avoiding damage to many healthy cells and simultaneously containing cellular metastasis.

In the monotonicity test, the accuracy of the PID control developed in Python and the performance of the power control board constructed to receive the PID information were evaluated. In this evaluation, it was verified that the percentages of output power errors measured by the oscilloscope in relation to those obtained by the ablation equipment at the critical points highlighted in Figure 5 are well below that specified by NBR-IEC 60601-2-2 (Associação..., 2013). Further, the declared loads were within $50-385 \Omega$, as defined in the design. These results prove that the impedance range, defined by the declared loads, satisfies the experimental data obtained by (Alba et al., 2011). They verified an impedance variation of $65-130 \Omega$ in experiments performed on an ex vivo liver. It is noteworthy that with the impedance increase during the burning process, the quality factor of the filter increases as demonstrated by its theory. Moreover, for impedances greater than $130 \Omega$, which result in quality factors greater than
0.713 at a $400 \mathrm{kHz}$-frequency, power peaks occur, and neuromuscular stimulation may occur; this indicates that muscle contractions may occur in a manner that could cause injury to the patient, as described in the theoretical part. This problem was solved by applying power controlled strictly by software.

The $\mathrm{CF}$ is fundamental in assessing the quality of the pure sine wave applied to the liver. If the amplitude of the sinusoidal signal is less than $200 \mathrm{~V}_{\mathrm{p}}$, the signal does not cut the tissue, but dissects and coagulates it. Further, regarding a $\mathrm{DC}$ voltage, it does not produce voltaic arcs and sparks that can cause burns (Schneider et al., 2007). The CF measurements found that the equipment output has a sinusoidal signal with very low distortion when applied to impedances greater than $50 \Omega$. The results show that for resistive loads above $50 \Omega$, the errors remain at approximately $2 \%$ compared to the pure theoretical sine wave (Figure 6), thereby indicating that there is a slight distortion in the equipment output. However, it does not influence the ablation performance.

Moreover, for resistive loads lower than $50 \Omega$, the errors can reach $12.37 \%$, thereby representing a distortion of the pure sine signal of approximately $10 \%$ higher compared to higher loads, Figure 6 . This distortion is expectable because the load decrease reduces the $Q_{\mathrm{p}}$ of the filters. The reduction implies that the poles shift away from the imaginary axis in the complex plane and, consequently, degrade the selectivity of the filter. This causes an amplitude amplification of the high frequency components leading to an increase of the $\mathrm{CF}$ error.

The evaluation of $Q_{\mathrm{p}}$ of the PI filter inserted at the frequency inverter output demonstrated that there was no increase in signal distortion with increasing load. This results in an increase of the filter selectivity up to the expected maximum threshold, as stated in the theory. Although, relatively high distortion occurs for impedances less than $50 \Omega$, the signal format remains sinusoidal and it is not possible to visually perceive any distortion on the oscilloscope screen.

The results showed that the equipment was able to fully comply with the NBR-IEC 60601-2-2 standard (Associação..., 2013). The thresholds were much higher than the recommended ones, thereby proving the technical capability of the equipment. However, there exists still need for new technical tests to verify the electromagnetic compatibility and the identification of the linear dynamic system to propose equipment improvements; for this, it is important to evaluate the dynamic characteristics in more detail. In addition, clinical trials are necessary to fully comply with the ANVISA recommendations (Agência..., 2017) and transform this prototype into a commercial product.

The promising results and the low material costs motivated the development of this equipment. The cost 
of the discrete electronics, inductors, transformers, and printed circuit boards made was approximately $\$ 742.00$. The high-impact polystyrene case (HIPS) for the circuits cost $\$ 303.00$, which leads to a total sum of $\$ 1,045.00$. Evidently, the prototyping costs are higher than the production costs.

\section{Acknowledgements}

The authors are grateful to the Ministério da Saúde (MS) - Brazil, for the financial support.

\section{References}

Agência Nacional de Vigilância Sanitária-ANVISA. Consultas [Internet]. Brasília: ANVISA; 2017. [cited 2017 Jul 25]. Available from: https://consultas.anvisa.gov.br

Alba J, González-Suárez A, Trujillo M, Berjano E. Theoretical and experimental study on RF tumor ablation with internally cooled electrodes: when does the roll-off occur? Conf Proc IEEE Eng Med Biol Soc. 2011; 2011:314-7. https://doi. org/10.1109/IEMBS.2011.6090082. PMID: 22254312.

Alexander C, Sadiku M. Fundamentos de circuitos elétricos. São Paulo: McGraw-Hill Companies Inc.; 2008.

Associação Brasileira de Normas Técnicas - ABNT. NBR-IEC 60601-2-2. Equipamento eletromédico, Parte 2: Requisitos particulares para a segurança básica e desempenho essencial de equipamentos cirúrgicos de alta frequência e acessórios cirúrgicos de alta frequência. Rio de Janeiro; 2013. 80 p.

Audigier C, Mansi T, Delingette H, Rapaka S, Mihalef V, Carnegie D, Boctor E, Choti M, Kamen A, Ayache N, Comaniciu, D. (2015). Efficient lattice boltzmann solver for patient-specific radiofrequency ablation of hepatic tumors. IEEE Transactions on Medical Imaging, 34(7), 1576-1589. http://dx.doi.org/10.1109/TMI.2015.2406575.

Bouza C, López-Cuadrado T, Alcázar R, Saz-Parkinson Z, Amate JM. Meta-analysis of percutaneous radiofrequency ablation versus ethanol injection in hepatocellular carcinoma. BMC Gastroenterol. 2009; 9:31.

Cambridge University Press. Cambridge dictionary [Internet]. Cambridge: Cambridge University Press; 2018. [cited 2018 Feb 25]. Available from: https://dictionary.cambridge.org/ $\mathrm{pt} /$ dicionario/ingles/ultrasound

Clavien, P., Lesurtel, M., Bossuyt, P., Gores, G., Langer, B., \& Perrier, A. Recommendations for liver transplantation for hepatocellular carcinoma: an international consensus conference report. Lancet Oncol. 2012; 13(1):e11-22. https:// doi.org/10.1016/S1470-2045(11)70175-9. PMID: 22047762.

Fairchild Semiconductor. LM78XX [Internet]. California: Fairchild Semiconductor; 2008. [cited 2018 Jan 1]. Available from: www.fairchildsemi.com

Frenzel LE Jr. Eletrônica moderna, fundamentos, dispositivos, circuitos e sistemas. São Paulo: McGraw Hill; 2015. p. 760-761.

Gomes MA, Priolli DG, Tralhão JG, Botelho MF. (2013). Hepatocellular carcinoma: epidemiology, biology, diagnosis and therapies. Rev Assoc Med Bras. 59(5), 514-24. https:// doi.org/10.1016/j.ramb.2013.03.005. PMID: 24041910.

Haemmerich D, Staelin ST, Tungjitkusolmun S, Lee FT Jr, Mahvi DM, Webster JG. Hepatic bipolar radio-frequency ablation between separated multiprong electrodes. IEEE Trans Biomed Eng. 2001; 48(10):1145-52. https://doi. org/10.1109/10.951517. PMID: 11585038 .

International Rectifier. IR2110 [Internet]. California: International Rectifier; 2005. [cited 2018 Jan 1]. Available from: www.irf.com

Kavak, F. A sizing algorithm for non-overlapping. Linköping, Suécia: Institutionen för systemteknik; 2004. p. 581-83.

Kikuchi LOO. (2015). Análise da sobrevida de pacientes com carcinoma hepatocelular atendidos no Instituto do Câncer do Estado de São Paulo [tese]. São Paulo: Universidade de São Paulo.

Linear Technology. LT1014 [Internet]. California: Linear Technology; 1990. [cited 2018 Jan 1]. Available from: www. linear.com

Nexperia. PCF8591 [Internet]. Nimega: Nexperia; 2013. [cited 2018 Jan 1]. Available from: www.nexperia.com

Nexperia. 74HC14 [Internet]. Nijmegen: Nexperia; 2017. [cited 2018 Jan 1]. Available from: www.nexperia.com

On Semiconductor. BC556 [Internet]. Colorado: On Semiconductor; 2007. [cited 2018 Jan 1]. Available from: www.onsemi.com

Philips Semiconductors. 74HC00 [Internet]. Amesterdã: Philips Semiconductors; 1997. [cited 2018 Jan 1]. Available from: www.semiconductors.philips.com

Pinheiro MM. Desenvolvimento de um eletrodo expansível de níquel-titânio para ablação hepática por radiofrequência Brasília: Universidade de Brasília; 2016.

Richman P. Wave factors for rectifiers with capacitor input filters, and other high crest-factor loads. IEEE Trans. Ind. Electron. Control Instrum. 2002; 21(4):235-41.

Schneider B, Bernardi R. Abatti PJ. A faísca eletrocirúrgica como causa da eletroestimulação e da queimadura DC. In: Müller-Karger C, Wong S, La Cruz A, editors. IV Latin American Congress on Biomedical Engineering 2007: Bioengineering Solutions for Latin America Health. IFMBE Proceedings; 2007 Sep 24-8; Berlin, Heidelberg. USA: Springer; 2007. p. 881-85.

Schneider-Junior B, Abatti PL. Desenvolvimento de um equipamento eletrocirúrgico com saída não chaveada. Revista Brasileira de Engenharia Biomédica; 2005; 21(1):15-24.

Texas Instruments. LM317 [Internet]. Dallas: Texas Instruments; 2017a. [cited 2018 Jan 1]. Available from: http://www.ti.com

Texas Instruments. LM324 [Internet]. Dallas: Texas Instruments; 2017b. [cited 2018 Jan 1]. Available from: http://www.ti.com

Texas Instruments. ADS1115 [Internet]. Dallas: Texas Instruments; 2018. [cited 2018 Jan 1]. Available from: http:// www.ti.com 
Thornton Eletrônica Ltda. Consultas [Internet]. São Paulo: Thornton; 2018. [cited 2018 Apr 5]. Available from: http:// www.thornton.com.br/home.htm

Vernetti JD. Introdução à teoria de redes. Manaus: ULBRA; 2003. p. 97-107.

Vishay Siliconix. IRF540 [Internet]. California: Vishay Siliconix; 2013a. [cited 2018 Jan 1]. Available from: www.vishay.com

Vishay Siliconix. 1N5408 [Internet]. California: Vishay Siliconix; 2013b. [cited 2018 Jan 1]. Available from: www.vishay.com
Xiaojing L, Wang Y, Zhu J, Guo Y, Gang L, Chengcheng L. Calculation of capacitance in high-frequency transformer windings. IEEE Transactions on Magnetics; 2016; 52(7):1-4. Zheng Q, Wu M. Evaluation of therapeutic effect of contrastenhanced ultrasonography in hepatic carcinoma radiofrequency ablation and comparison with conventional ultrasonography and enhanced computed tomography. Journal of Medical Ultrasound; 2015; 23:76-81. 